\title{
Tuberculosis Characterization in a Special Population of Kidney Transplant Recipients
}

\author{
Barbara Reis-Santos ${ }^{1}$ and Ethel Leonor Noia Maciel ${ }^{1,2,3}$ \\ ${ }^{1}$ Programa de Pós-graduação em Doenças Infecciosas, Centro de Ciências da Saúde, Universidade Federal do Espírito Santo, \\ Vitória, ES, Brazil \\ ${ }^{2}$ Programa de Pós-graduação em Saúde Coletiva, Centro de Ciências da Saúde, Universidade Federal do Espírito Santo, \\ Vitória, ES, Brazil \\ ${ }^{3}$ Laboratório de Epidemiologia, Avenue, Marechal Campos, 1468, Maruípe, Vitória, ES, Brazil
}

Correspondence should be addressed to Ethel Leonor Noia Maciel; ethel.maciel@gmail.com

Received 7 August 2012; Accepted 23 August 2012

Academic Editors: D. Bachani, D.-D. Ji, T. Matsumoto, and D. Nardelli

Copyright (C) 2013 B. Reis-Santos and E. L. N. Maciel. This is an open access article distributed under the Creative Commons Attribution License, which permits unrestricted use, distribution, and reproduction in any medium, provided the original work is properly cited.

Setting. Tuberculosis clinical presentation is not typical in kidney transplant recipients and the diagnosis of active disease is usually delayed. Objective. To characterize tuberculosis presentation in Brazilian's kidney transplant recipients. Study Design. We analyzed the clinical records of tuberculosis cases regarding sociodemographic data and health history. Results. Thirteen TB cases were identified among 843 transplant recipients. The average time for TB development after transplantation was 4 years. Eight subjects presented pulmonary disease, seven patients required hospitalization Alertness to the possibility of TB and the careful evaluation for possible TB of all kidney transplant recipients with unexplained is vital, as is the related work of transplant and TB control teams and four died as a consequence of TB. Conclusion. The severe consequences of TB in posttransplantation can become critical.

\section{Introduction}

Worldwide public health programs agree that tuberculosis (TB) remains a major challenge. Several national and international organizations have worked together for TB control, and estimates of TB incidence, prevalence, and mortality are improving. TB prevalence in 2010 was estimated at 178 cases per 100.000 people (95\% CI, 156-201) worldwide [1].

Although substantial progress has been made, some conditions, especially related to noncommunicable diseases, still hinder the ultimate control of tuberculosis $[2,3]$. Chronic kidney disease is one of these, and in kidney transplant subjects $T B$ is an important opportunistic infection. Its frequency can be up 30 times greater among transplant recipients than in the general population [4].

Kidney transplant subjects generally have reactivation of latent infections as the most common form of tuberculosis development and due to the clinical presentation of TB is not characteristic, with more extrapulmonary events and nonspecific symptoms, the diagnosis of active disease is usually delayed [5].

Mortality by TB in kidney transplant population is the highest among subjects with posttransplant $\mathrm{TB}$, and kidney is the most frequently transplanted organ [6]. The graft function can be compromised by both the direct effects of tuberculosis as a drug interactions, which complexes the management of those subjects $[7,8]$.

In the Brazilian state of Espírito Santo (ES), about 1,500 kidney transplants have been performed in 10 transplantation centers of their past 35 years. Thus, this study aims to characterize tuberculosis presentation in Brazilian's kidney transplant recipients.

\section{Patients and Methods}

This study included all thirteen [9] cases of tuberculosis identified among 843 kidney transplant recipients whose records were available. These records were analyzed retrospectively 
TABLE 1: Clinical features of kidney transplant recipients with tuberculosis.

\begin{tabular}{lcccccccc}
\hline Patient & Gender & Previous TB & Latent TB treatment & TST & X-ray alteration & DX & Type disease & Outcome \\
\hline 01 & Female & Yes & No & Positive & Yes & AARB & Pulmonary & Cured \\
02 & Female & No & No & Positive & Yes & AARB & Pulmonary & Cured \\
03 & Female & No & No & Negative & No & Culture & Pulmonary & Cured \\
04 & Male & No & N/D & Positive & No & Clinical & Extrapulmonary & Died \\
05 & Female & No & N/D & Positive & Yes & AARB & Pulmonary & Died \\
06 & Male & N/D & N/D & Positive & No & Clinical & Extrapulmonary Disseminated \\
07 & Male & No & No & Negative & No & Hist. ex. & Pulmonary & Cured \\
08 & Male & No & No & Negative & No & Hist. ex. Extrapulmonary & Cured \\
09 & Male & No & No & Positive & Yes & AARB & Pulmonary & Cured \\
10 & Male & Yes & Yes & Positive & Yes & AARB & Disseminated & Died \\
11 & Male & No & No & Positive & Yes & Hist. ex. Extrapulmonary & Cured \\
12 & Female & No & No & Negative & No & Culture & Pulmonary & Cured \\
13 & Male & No & No & Positive & Yes & AARB & Pulmonary \\
\hline
\end{tabular}

TB: tuberculosis; TST: tuberculin skin test; DX: diagnosis; AARB: demonstration of acid-alcohol resistant bacilli; Hist. ex.: histopathologic examination; N/D: not done.

with data collection following a structured guide with sociodemographic data, health history, chronic kidney disease, kidney transplantation, and tuberculosis being considered.

The sociodemographic covariates analyzed were age (years), gender (male/female), race (whites/non-whites), years of schooling ( $\leq 7$ years/ $>7$ years), marital status (married/nonmarried), and location of residence. The covariates related to history of health were type of donor (live/deceased), time of renal replacement therapy (years), previous TB (no/yes), treatment of latent TB (no/yes), tuberculin skin test (negative/positive), X-ray alteration (no/yes), method of TB diagnosis (acid-alcohol resistant bacilli/culture/histopathologic examination/clinical), type of disease (pulmonary/extrapulmonary/disseminated), and TB treatment outcome (cured/ died/disseminated).

Information was structured and stored on an electronic database. After exploratory analysis, the data was evaluated for absolute and relative frequencies, mean, median, and standard deviations. Kaplan-Meier analysis was performed to construct survival plot of time of TB after kidney transplantation. These analyses were performed with Stata, version 12.0.

The study was approved by the ethics committee of Centro de Ciências da Saúde (Center of Health Sciences) of Universidade Federal do Espírito Santo-no. 204/10.

\section{Results}

Thirteen cases of tuberculosis were identified among 843 Brazilian kidney transplant recipients between June 1976 and May 2011. The mean age was $34( \pm 12)$ years old. $54 \%$ were nonwhites, $40 \%$ were married, $63 \%$ studied less than 7 years, and all lived in the capital of ES, Vitória, or its surroundings.

$67 \%$ of the transplants used deceased donors and were performed after $4.3( \pm 2.6)$ years of renal replacement therapy. The median for development of TB after the transplantation was 4 years and the survival estimate is shown in Figure 1.



Figure 1

The primary characteristics of tuberculosis are in Table 1, where we found that two subjects had a previous history of TB and only one received treatment for latent TB. Eight subjects presented with pulmonary disease and four died as a consequence of TB.

The subjects were treated in ambulatory units or reference hospitals of transplant programs. Twelve patients received a traditional antituberculosis regimen which until 2010 consisted of the rifampicin, isoniazid, and pyrazinamide. In 2010, ethambutol was added to the regimen, as recommended by Brazilian Ministry of Health [10]. Even with this treatment regimen, seven required hospitalization.

\section{Discussion}

Secondary data has some limitations due to loss of information. However we believe that we managed to minimize this difficulty through the crossover between the database of the study and the national registry of tuberculosis (SINAN). 
Kidney transplant recipients have a high risk for developing primary infections as well as reactivation of latent infections from Mycobacterium tuberculosis [11]. Factors associated with an even greater increase in risk for TB include the time of renal replacement therapy, previous transplantation, immunosuppressive regimen, and the number of rejection episodes. Despite its upper limit in the first year after transplant, we found that half the sample developed TB within 4 years after procedure $[5,12]$.

The diagnosis of TB in transplant subjects is usually delayed. This delay occurs because the clinical presentation of TB does not usualy mean unexpected. The extrapulmonary and disseminated forms and nonspecific symptoms are more frequent than those of the general population and we verified this trend. Thus, the traditional diagnostic methods such as $\mathrm{X}$-ray, demonstration of acid-alcohol resistant bacilli in body fluids smears, and growth of TB bacilli in culture specimens are unable to identify the subjects which also appear in our sample $[5,11,13]$.

Tuberculin skin test is widespread for TB screening but the immunosuppression used after transplantation interferes with the test accuracy where a positive test is useful but a negative test does not exclude the disease [5,9]. The four subjects with negative TST fit into this context.

Although the recommendation of TB treatment in kidney transplant recipients follows the traditional scheme, controversies remain. Some drugs for TB treatment are nephrotoxic and can lead to a negative outcome either for transplantation or for TB treatment [13-15]. High fever and erythema occurred upon resumption of isoniazid; however, the symptoms gradually improved with oral prednisolone after cessation of isoniazid and transplant recipients generally took this corticoid routinely for graft support [5].

\section{Conclusion}

Consideration of the severe consequences of $\mathrm{TB}$ in posttransplantation period makes clear the crucial importance of the careful evaluation of all kidney transplant recipients with unexplained symptoms for TB. The treatment of these subjects is difficult and must be closely monitored. The challenges that TB presents in connection with transplant subjects will be overcome only when TB and transplantations teams work together for the best care of this special population.

\section{Funding}

Edital MCT/CNPq/MS and ICOHRTA 5 U2RTW006883-02.

\section{References}

[1] M. Raviglione, B. Marais, K. Floyd et al., "Scaling up interventions to achieve global tuberculosis control: progress and new developments," The Lancet, vol. 379, no. 9829, pp. 1902-1913, 2012.

[2] S. D. Lawn and A. I. Zumla, "Tuberculosis", The Lancet, vol. 378, no. 9785, pp. 57-72, 2011.

[3] G. Ferrara, M. Murray, K. Winthrop et al., "Risk factors associated with pulmonary tuberculosis: smoking, diabetes and
anti-TNFalpha drugs," Current Opinion in Pulmonary Medicine, vol. 18, no. 3, pp. 233-240, 2012.

[4] B. Reis-Santos, T. Gomes, B. L. Horta, and E. L. N. Maciel, "Prevalência de tuberculoseentre transplantados renais: revisão Sistemática e Meta-Análise," Revista de Saúde Pública. In press.

[5] H. Milburn, N. Ashman, P. Davies et al., "Guidelines for the prevention and management of Mycobacterium tuberculosis infection and disease in adult patients with chronic kidney disease," Thorax, vol. 65, no. 6, pp. 559-570, 2010.

[6] N. Singh and D. L. Paterson, "Mycobacterium tuberculosis infection in solid-organ transplant recipients: impact and implications for management," Clinical Infectious Diseases, vol. 27, no. 5, pp. 1266-1277, 1998.

[7] R. Ram, G. Swarnalatha, N. Prasad, and K. V. Dakshinamurty, "Tuberculosis in renal transplant recipients," Transplant Infectious Disease, vol. 9, no. 2, pp. 97-101, 2007.

[8] A. C. Currie, S. R. Knight, and P. J. Morris, "Tuberculosis in renal transplant recipients: the evidence for prophylaxis," Transplantation, vol. 90, no. 7, pp. 695-704, 2010.

[9] P. A. Triverio, P. O. Bridevaux, P. Roux-Lombard et al., "Interferon-gamma release assays versus tuberculin skin testing for detection of latent tuberculosis in chronic haemodialysis patients," Nephrology Dialysis Transplantation, vol. 24, no. 6, pp. 1952-1956, 2009.

[10] M. B. Conde, F. A. Melo, A. M. Marques et al., "III Brazilian thoracic association guidelines on tuberculosis," The Jornal Brasileiro de Pneumologia, vol. 35, no. 10, pp. 1018-1048, 2009.

[11] V. Lezaic, V. Lezaic, R. Radivojevic et al., "Does tuberculosis after kidney transplantation follow the trend of tuberculosis in general population?" Renal Failure, vol. 23, no. 1, pp. 97-106, 2001.

[12] X. F. Zhang, Y. Lv, W. J. Xue et al., "Mycobacterium tuberculosis infection in solid organ transplant recipients: experience from a single center in China," Transplantation Proceedings, vol. 40, no. 5, pp. 1382-1385, 2008.

[13] A. K. Subramanian and E. L. Nuermberger, "Tuberculosis in transplant recipients: diagnostic and therapeutic dilemmas," Transplant Infectious Disease, vol. 10, no. 4, pp. 229-230, 2008.

[14] M. R. Holdiness, "Clinical pharmacokinetics of the antituberculosis drugs," Clinical Pharmacokinetics, vol. 9, no. 6, pp. 511-544, 1984.

[15] F. A. Manian, W. J. Stone, and R. H. Alford, "Adverse antibiotic effects associated with renal insufficiency," Reviews of Infectious Diseases, vol. 12, no. 2, pp. 236-249, 1990. 


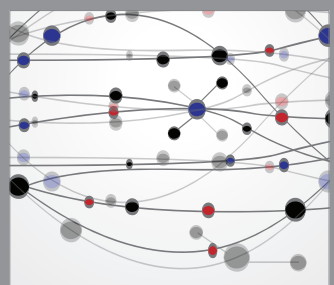

The Scientific World Journal


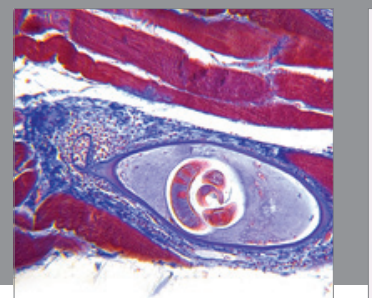

Gastroenterology

Research and Practice
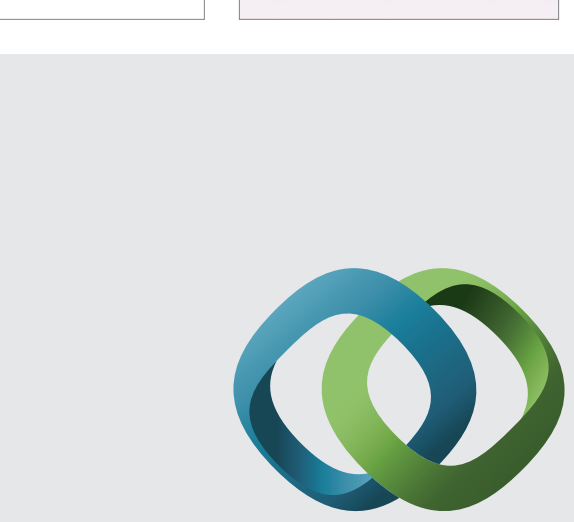

\section{Hindawi}

Submit your manuscripts at

http://www.hindawi.com
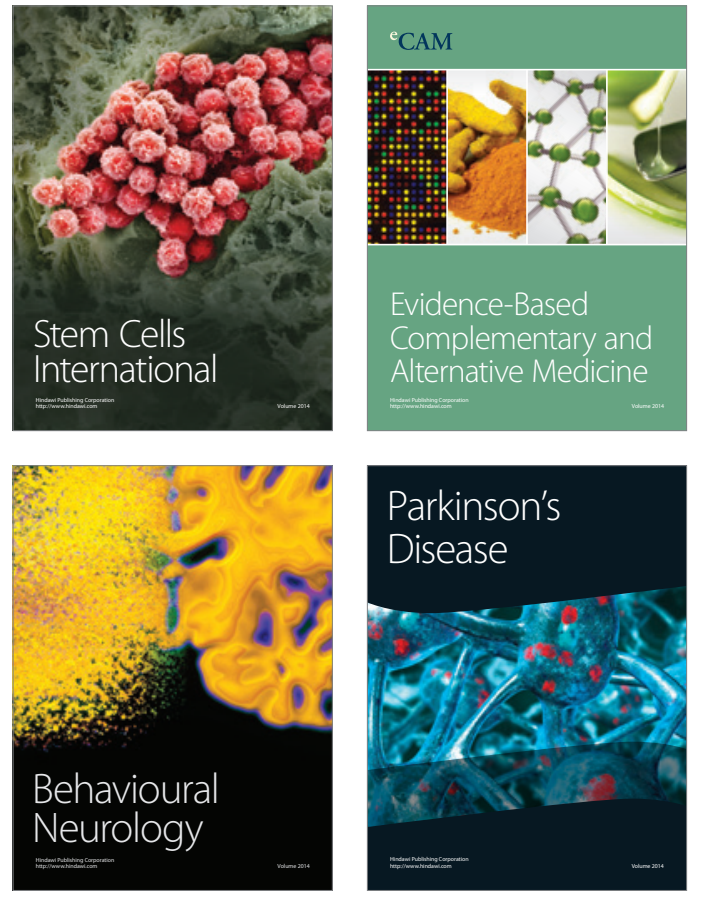


Disease Markers
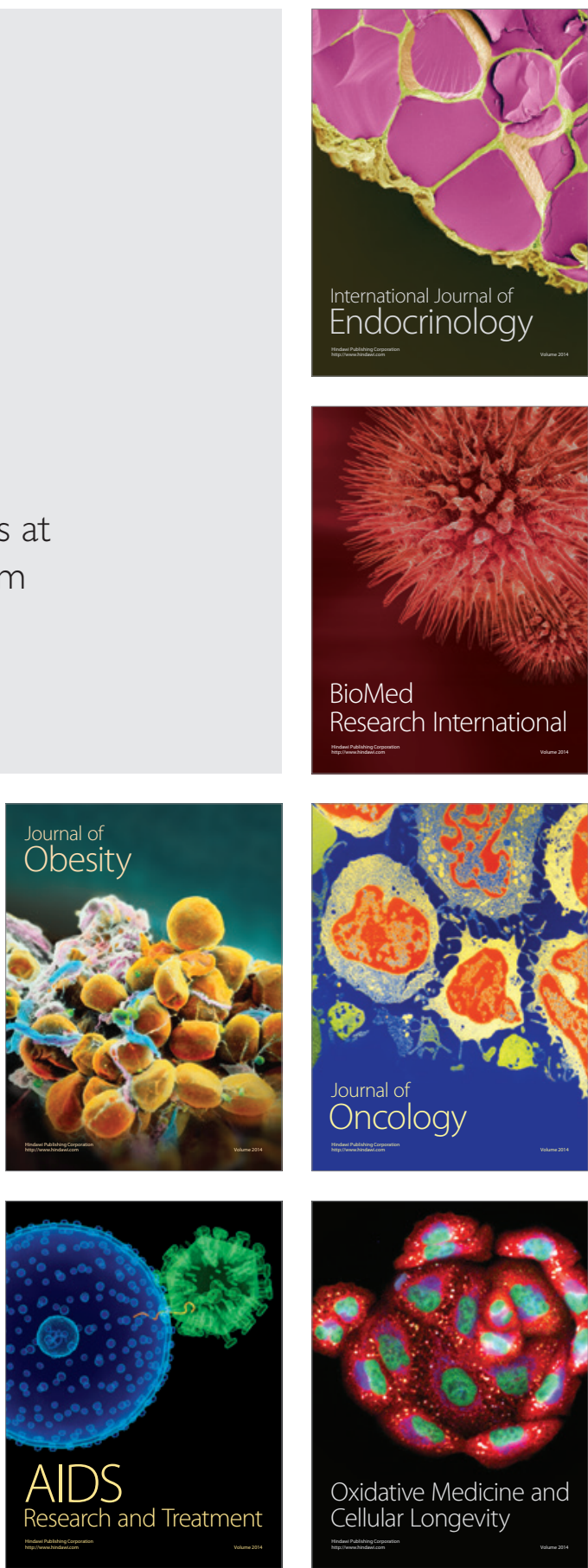